\title{
Two-stage Total Hip Arthroplasty
}

\section{How Often Does It Control Methicillin-resistant Infection?}

\author{
Fay Leung MD, FRCSC, Corey J. Richards MD, MASc, FRCSC, \\ Donald S. Garbuz MD, MHSc, FRCSC, \\ Bassam A. Masri MD, FRCSC, Clive P. Duncan MB, MSc, FRCSC
}

Published online: 16 December 2010

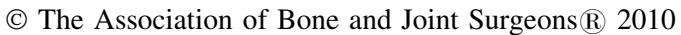

\begin{abstract}
Background Methicillin-resistant hip infections are increasingly common. Reports of the surgical management of these patients using two-stage THA show variable control of infection, but all reports used static spacers. Questions/purposes We therefore determined (1) the rate of successful control of infection and (2) function in patients with methicillin-resistant infection treated with a two-stage THA using an articulated cement spacer during the first stage.

Methods We retrospectively reviewed 50 patients who had a two-stage revision THA for methicillin-resistant Staphylococcus aureus or methicillin-resistant Staphylococcus epidermidis infection. Twelve patients died, leaving 38 for review. All eligible patients completed quality-oflife outcome questionnaires (WOMAC, SF-12, Oxford-12, UCLA activity score, hip and knee satisfaction score).
\end{abstract}

Each author certifies that he or she has no commercial associations
(eg, consultancies, stock ownership, equity interest, patent/licensing
arrangements, etc) that might pose a conflict of interest in connection
with the submitted article.
Each author certifies that his or her institution approved the human
protocol for this investigation, that all investigations were conducted
in conformity with ethical principles of research, and that informed
consent for participation in the study was obtained.
This work was performed at the Division of Lower Limb
Reconstruction and Oncology, Department of Orthopaedics,
University of British Columbia.

F. Leung, D. S. Garbuz, B. A. Masri, C. P. Duncan ( $\square)$ Department of Orthopaedics, University of British Columbia, 3114-910 West 10th Avenue, Vancouver, BC V5Z 4E3, Canada e-mail: clive.duncan@vch.ca; cliveduncan@hotmail.com

C. J. Richards

Department of Orthopaedics, Hull Hospital,

Gatineau, QC, Canada
Minimum followup was 24 months after the second stage (mean, 58 months; range, 24-123 months).

Results Of the 38 patients, eight (21\%) had recurrence of their infection requiring further revision surgery. Of the remaining 27 patients, the mean WOMAC was 62 , mean Oxford-12 60, mean UCLA activity score 4.3, and mean hip and knee satisfaction score 66.

Conclusions We found a treatment failure rate of $21 \%$ for patients with methicillin-resistant S. aureus or methicillinresistant $\mathrm{S}$. epidermidis infection. This is a higher rate than reported for two-stage THA for studies including patients infected with both nonresistant and resistant organisms. The functional scores for patients were also lower than those reported in the literature.

Level of Evidence Level IV, therapeutic study. See Guidelines for Authors for a complete description of levels of evidence.

\section{Introduction}

The prevalence of infection after THA is reportedly between $1 \%$ and $2 \%$ for primary THA and between $3 \%$ and $4 \%$ for revision THA [9]. Multiple treatment options exist for the management of an infected THA, including longterm antibiotic suppression $[6,10]$, surgical débridement [6], one-stage [4, 5] or two-stage exchange [11, 12, 17, 19], excision arthroplasty [13], arthrodesis [16], and amputation. Our preferred management of a chronically infected THA, when patient factors allow, is a two-stage revision THA. The use of an articulated antibiotic-loaded spacer allows for maintenance of soft tissue tension and patient function between stages. Previous studies with this type of prosthesis have reported control of infection in $89 \%$ to $96 \%$ of patients $[8,19,28]$. 
In the last decade, the prevalence of infections caused by resistant organisms such as methicillin-resistant Staphylococcus aureus (MRSA) and methicillin-resistant Staphylococcus epidermidis (MRSE) has increased [23]. The reported successful control of infection after the surgical management of these patients with a two-stage THA is quire variable, with success rates ranging from $47 \%$ to $100 \%[15,20,25]$. To our knowledge, there are no studies to date reporting on the success rate for control of methicillin-resistant infection in patients managed with twostage THA using an articulated cement spacer during the first stage.

We therefore determined (1) the rate of successful control of infection and (2) function in patients with methicillin-resistant infection treated with a two-stage THA using an articulated cement spacer during the first stage.

\section{Patients and Methods}

We retrospectively reviewed the medical records of 50 patients treated with a two-stage THA using the PROSTALAC $^{\circledR}$ (DePuy, Warsaw, IN) articulated spacer for a methicillin-resistant hip infection (minimum two of three specimens on preoperative hip aspirate and/or intraoperative cultures) between January 1998 and March 2006. There were 29 men and 21 women. Three of the 50 patients were excluded because they died from nonassociated medical conditions before receiving their second stage. Nine patients died an average of 4 years (range, 2-10 years) after the second stage of treatment, leaving 38 patients available for analysis. The diagnosis at the time of death for the nine patients was not related to sepsis or infection, although only three patients had undergone blood work to rule out infection and none of the patients had undergone hip aspiration to confirm absence of hip infection. The average age at the time of diagnosis and initial treatment was 63.5 years (range, 36-80 years). Of the 38 patients, 20 had an infected primary THA, 11 an infected revision THA, and seven infected internal fixation devices for prior proximal femur or acetabular fracture. The minimum followup was 24 months after the second stage (mean, 58 months; range, 24-123 months). None of the 38 patients was lost to followup. Institutional Review Board approval was obtained to enroll these patients in this study.

The causative organisms were MRSA in 10 patients, MRSE in 26, and both MRSA and MRSE in two. Nine patients had multiple organisms isolated (MRSA and methicillin-sensitive $\mathrm{S}$. aureus, MRSA and Group B Streptococcus, MRSA and Candida albicans, MRSA and Proprionibacterium, MRSA and Enterococcus faecalis,
MRSA and Enterococcus faecalis and Peptostreptococcus, MRSE and Staphylococcus lugdunensis, MRSE and Klebsiella pneumoniae, MRSE and Propriobacteria).

All patients who were referred to our tertiary-care arthroplasty center with a suspected chronic hip infection were evaluated preoperatively through a standard workup, including a thorough history, physical examination, erythrocyte sedimentation rate (ESR), C-reactive protein (CRP), and aspiration of the hip. All patients had a culture-positive bacterial infection with MRSE or MRSA and elevated ESR $(>30)$ and CRP $(>10)$.

All patients were treated under the care of the four specialized adult reconstructive surgeons (DSG, BAM, CPD, NVG) at our institution who have a subspecialized interest in the management of infected joint arthroplasty. Two-stage THA using the PROSTALAC ${ }^{\circledR}$ articulated spacer was performed as previously described $[8,19,28]$. The first stage consisted of removal of infected implants, hardware, and cement followed by meticulous débridement of all necrotic and infected tissue. Pseudocapsule, acetabular, and femoral canal membranous tissue were removed and a minimum of three specimens sent for culture and sensitivity evaluation. The operative site was then thoroughly irrigated with a large volume of normal saline. An appropriately sized PROSTALAC ${ }^{\circledR}$ femoral component was then fashioned using antibiotic-loaded Palacos ${ }^{\circledR}$ cement (Zimmer, Inc, Warsaw, IN). The acetabular component is a one-size-fits-all cup (42-mm outside diameter, 32-mm inside diameter) with a snap-fit configuration fixed in place using antibiotic-loaded cement. The appropriate antibiotic powder was added to the cement powder based on the sensitivity of the infecting organism. In most cases (29 of 38), a combination of $1.5 \mathrm{~g}$ vancomycin and $3.6 \mathrm{~g}$ tobramycin was added per $40 \mathrm{~g}$ cement. In a smaller number (nine of 38), the antibiotic was added to Palacos ${ }^{\circledR}$ cement already containing $0.5 \mathrm{~g}$ gentamicin. The femoral component, consisting of a simple metal endoskeleton and modular head-neck junction, was encased in a mantle of antibiotic-loaded cement preset within a mold of suitable size. The mold sizes match a set of broaches used to prepare and size the femoral canal. This composite was pressfitted into the canal. In some cases of severe proximal bone loss, a supplementary collar of antibiotic cement was added to enhance rotational stability.

Postoperatively, patients were instructed to $50 \%$ partially weightbear and treated with a 6-week course of intravenous or combined intravenous and oral antibiotics in consultation with an infectious disease consultant. A physiotherapist assessed all patients Postoperative Day 1 and immediately began supervised ROM exercises and weightbearing exercises. Patients initially mobilized using a walker and progressed, if able, to crutches. Physiotherapy was provided throughout the hospital stay and continued on 
an outpatient basis once the patient was discharged from the hospital. All patients were treated with intravenous vancomycin between stages with ciprofloxacin added to the regimen in three patients and rifampin in eight. Oral rifampin was added to the postoperative regimen later in the series (the final eight patients) in an attempt to better control resistant infections. The addition of intravenous ciprofloxacin was at the discretion of the consulting infectious disease specialist.

All patients were assessed at a minimum of 8 weeks postoperatively ( 2 weeks after cessation of all antibiotics). During this followup visit, patients underwent a thorough history and physical examination focusing on evidence of recurrence of infection. In addition, hip radiographs were taken and blood work (ESR and CRP) was performed. Hip aspirates (performed on eight of 38 patients) were subsequently undertaken to rule out persistent infection when deemed necessary (clinical signs of infection or elevated in laboratory studies). Six patients underwent repeat operations before their second stage; four patients required a repeat first stage for persistent infection confirmed with hip aspiration; one patient underwent irrigation and débridement shortly after the first stage for evacuation of an aseptic hematoma; and one additional patient had a revision of firststage PROSTALAC ${ }^{\circledR}$ for an aseptic dislocation. Patients were scheduled for the second-stage revision THA once there was no clinical evidence of infection and inflammatory markers (ESR and CRP) had demonstrated a steady decline or had already decreased to normal limits a minimum of 2 weeks after the cessation of antibiotics. Equivocal cases were evaluated with hip aspirations; those patients with a positive result underwent repeat first-stage surgery; and those patients with a negative culture were scheduled for a definitive second-stage procedure.

The mean time between the first and second stage was 6 months (range, 2-15 months). The typical protocol was 6 weeks antibiotics followed by repeat investigation after another 4 weeks (total 10) and a scheduled intervention 2 weeks later (12 weeks after the first stage). For logistic reasons relating to waiting list difficulties, patient preference, optimization of medical condition, and others, there was a wide variation in the actual date of readmission for the second-stage procedure. At the second stage, the PROSTALAC $^{\circledR}$ system was removed and the hip was reconstructed with appropriate cementless revision implants. A frozen section was not carried out during the second stage because, in our view, the sensitivity and specificity of this adjunct had not been established in this second-stage setting within the time frame of this study. Intravenous antibiotics were continued until the final intraoperative culture results were available, usually at 5 days. Intraoperative cultures during the second-stage procedure were negative in the 38 study patients.
Each patient underwent a similar postoperative rehabilitation regime after the first stage with the exception that weightbearing restrictions were either nonweightbearing or $50 \%$ weightbearing as decided by the operating surgeon based on the surgical reconstruction, intraoperative findings, and postoperative radiographs.

All patients were then followed 6 weeks postoperatively and then yearly thereafter. A thorough history, physical examination, and standard hip radiographs were undertaken at each visit. Patients with either clinical or radiographic findings suspicious for infection underwent laboratory studies, including ESR and CRP.

The primary outcome of our study was the success rate for control of infection after two-stage hip arthroplasty. The medical charts of all 38 patients were retrospectively reviewed to ascertain those who had documented evidence of recurrence of infection. The remaining patients were mailed a questionnaire regarding recurrence of infection. Four questions were asked: (1) "Have you had any further episodes of infection in the hip replacement requiring investigation?" (2) "Have you had any drainage or wound breakdown on the operated hip requiring investigation?" (3) "Have you had any further surgery on the operated hip?" (4) "Are you awaiting any further surgery on the operated hip?" Those patients who did not respond to the mailed questionnaires were contacted by telephone. Two patients were lost to followup. Patients with negative hip aspirations and those who answered no to all four questions were deemed to have adequate control of infection. No patients were on suppressive antibiotics at the time of assessment. Information regarding previous surgical procedures, infecting organisms, antibiotic regimen, comorbidities, and laboratory data was collected from medical charts. Comorbidities and the number of prior surgeries were evaluated as potential risk factors for treatment failure. The comorbidities reviewed included diabetes, exogenous steroid use, hepatitis $\mathrm{C}$ infection, renal insufficiency, obesity, and smoking history.

The patients who had not undergone a revision for recurrence of infection were mailed quality-of-life outcome questionnaires to assess functional outcomes. Those patients who did not respond were then contacted by telephone to complete the questionnaires. The quality-oflife questionnaire included the WOMAC osteoarthritis index [2], Oxford-12 [7], SF-12 [26], UCLA activity scales [1], and the hip and knee arthroplasty satisfaction questionnaire [14]. We normalized each raw score into a 0 to 100 scale with 0 being the worst quality of life and 100 the best quality of life.

We compared a number of categorical variables against success/failure using Fisher's exact test for the dichotomous variables and Pearson's chi square test for the multicategorical variables. The continuous variables age at 
surgery and body mass index were analyzed in univariate logistic regression models predicting control of infection.

\section{Results}

At last followup, infection had been controlled in 30 of 38 patients $(79 \%)$, with eight patients having recurrence of infection with the same organism. One additional patient became infected with a new organism, Group B Streptococcus, which was managed with long-term suppressive oral antibiotics. This patient was not categorized as a treatment failure given that the initial infection was successfully controlled. Of the patients who had recurrent methicillin-resistant infection, two were female and six were male. The original organisms were MRSE in four patients, MRSE and mixed organisms in two patients, and MRSA and mixed organisms in two patients. Two of the eight patients with recurrent MRSE infection underwent a successful repeat two-stage reconstruction with eventual control of their infection at the time of last followup. All six remaining patients who ultimately failed treatment with a two-stage THA eventually required resection arthroplasty. Two of these six patients underwent a repeat

Table 1. Results of analysis of risk factors for success/failure of control of infection after two-stage THA using an articulated cement spacer

\begin{tabular}{|c|c|c|c|}
\hline \multirow[t]{2}{*}{ Variable } & \multicolumn{2}{|l|}{ Control of infection } & \multirow[t]{2}{*}{$\mathrm{p}$ Value } \\
\hline & Success $(\mathrm{n}=30 / 38 ; 78.95 \%)$ & Failure $(\mathrm{n}=8 / 38 ; 21.05 \%)$ & \\
\hline Mean age (years) & $62.07(\mathrm{SD}=12.26)$ & $65.12(\mathrm{SD}=11.86)$ & 0.5221 \\
\hline Mean body mass index $\left(\mathrm{kg} / \mathrm{m}^{2}\right)$ & $30.97(\mathrm{SD}=6.62)$ & $30.35(\mathrm{SD}=5.83)$ & 0.806 \\
\hline Gender & & & 0.2384 \\
\hline Male & $14(46.67 \%)$ & $6(75.00 \%)$ & \\
\hline Female & $16(53.33 \%)$ & $2(25.00 \%)$ & \\
\hline Infecting organism & & & 0.1903 \\
\hline MRSA & $3(10.00 \%)$ & $1(12.50 \%)$ & \\
\hline MRSE & $18(60.00 \%)$ & $2(25.00 \%)$ & \\
\hline Polymicrobial & $9(30.00 \%)$ & $5(62.50 \%)$ & \\
\hline Primary diagnosis & & & 0.8871 \\
\hline Osteoarthritis & $17(56.67 \%)$ & $5(62.50 \%)$ & \\
\hline Avascular necrosis & $5(16.67 \%)$ & $1(12.50 \%)$ & \\
\hline Inflammatory arthropathy & $2(6.67 \%)$ & & \\
\hline Fracture & $5(16.67 \%)$ & $2(25.00 \%)$ & \\
\hline Chondrosarcoma & $1(3.33 \%)$ & & \\
\hline Multiple previous surgeries & $12(40.00 \%)$ & $3(37.50 \%)$ & 1 \\
\hline Antibiotics used in cement & & & 0.3631 \\
\hline Vancomycin + tobramycin & $24(80.00 \%)$ & $5(62.50 \%)$ & \\
\hline Vancomycin + tobramycin + gentamicin & $6(20.00 \%)$ & $3(37.50 \%)$ & \\
\hline Antibiotics used after first stage & & & 0.7238 \\
\hline Intravenous vancomycin & $20(66.67 \%)$ & $6(75.00 \%)$ & \\
\hline Intravenous vancomycin + cipromycin & $1(3.33 \%)$ & $1(12.50 \%)$ & \\
\hline Intravenous vancomycin + oral rifampin & $6(20.00 \%)$ & $1(12.50 \%)$ & \\
\hline Intravenous vancomycin + gentamicin & $1(3.33 \%)$ & & \\
\hline Intravenous vancomycin + cloxacillin & $2(6.67 \%)$ & & \\
\hline \multicolumn{4}{|l|}{ Comorbidities } \\
\hline Diabetes & $4(13.33 \%)$ & $3(37.50 \%)$ & 0.1461 \\
\hline Steroid/immunosuppression therapy & $4(13.33 \%)$ & $1(12.50 \%)$ & 1 \\
\hline Chronic renal failure & $1(3.33 \%)$ & $1(12.50 \%)$ & 0.3812 \\
\hline Heart disease & $4(13.33 \%)$ & $2(25.00 \%)$ & 0.587 \\
\hline Lung disease & $5(16.67 \%)$ & $1(12.50 \%)$ & 1 \\
\hline Thyroid disease & $3(10.00 \%)$ & & 1 \\
\hline Smoking & $3(10.00 \%)$ & $2(25.00 \%)$ & 0.2793 \\
\hline
\end{tabular}

MRSA = methicillin-resistant Staphylococcus aureus; MRSE $=$ methicillin-resistant Staphylococcus epidermidis. 
Table 2. Quality-of-life outcome scores for patients with successful control of infection after two-stage THA using an articulated spacer

\begin{tabular}{ll}
\hline Quality-of-life measure* & Mean score \\
\hline Oxford hip score & 59.6 \\
SF-12 mental component & 47.9 \\
SF-12 physical component & 32.4 \\
Mean satisfaction score & 65.7 \\
WOMAC function & 59.5 \\
WOMAC pain & 67.1 \\
WOMAC stiffness & 64.3 \\
WOMAC global & 61.5 \\
UCLA activity score & 4.3
\end{tabular}

* Oxford hip score and WOMAC scores are normalized to a range of 0 to 100 with 0 being worst and 100 being best.

first-stage PROSTALAC ${ }^{\circledR}$ for continued signs of active infection that was unsuccessful and then subsequently required resection arthroplasty. Therefore, repeat two-stage THA was attempted on four of the eight initial patients who had failed treatment with a success rate of 50\% (two of four). The remaining four patients underwent resection arthroplasty after the diagnosis of the recurrence of infection. None of the variables analyzed as predictors of control of infection (Table 1) was significant (all $p$ values $>0.01$ )

Of the 27 patients with initial infection control for whom we had data, the mean WOMAC was 62 , mean Oxford-12 60, mean UCLA activity score 4.3, and mean hip and knee arthroplasty satisfaction score 66 (Table 2).

\section{Discussion}

Given the increasing prevalence of methicillin-resistant infection, the management of patients undergoing THA who are infected with these organisms is a growing concern. Reports of the surgical management of these patients using two-stage THA show control of infection ranging from $89 \%$ to $96 \%$ of patients $[8,19,28]$. These studies all reported use of static spacers. We therefore determined (1) the rate of successful control of infection and (2) function in patients with methicillin-resistant infection treated with a two-stage THA using an articulated cement spacer during the first stage.

We draw the reader's attention to the study limitations. First, it is a small series of patients, which therefore makes it difficult to draw definitive conclusions regarding the influence of the multitude of preoperative variables on success rate of treatment. Second, functional scores were only available on those patients who had successful control of their infection. The data therefore do not give a true assessment of the resulting functional outcomes of all patients undergoing a two-stage THA for methicillinresistant infection.

We were able to control the infection in 30 of 38 patients (79\%) using a two-stage THA when the infecting organism was a methicillin-resistant Staphylococcus. This rate is similar to that found by Parvizi et al. [20] who reported a 75\% successful control of infection when managing methicillin-resistant infection using a two-stage THA with an antibiotic cement spacer used during the first stage for 66 patients (Table 3). This rate is lower, however, compared to those reported by Volin et al. [25] and Toulson et al. [24], who both reported a $100 \%$ success rate in the management of resistant infection for six and 21 infected hip patients, respectively. When compared with a cohort of patients with both nonresistant and resistant infections managed using a two-stage reconstruction with an articulated spacer, our infection control rate was worse. Previous studies using the PROSTALAC ${ }^{\circledR}$ system reported outcomes on 28 patients infected with both resistant and nonresistant organisms who underwent reconstruction for infected THA and a reported success rate of 96\% [28]. A more recent study evaluating long-term outcomes of 103 patients with infected THAs involving both resistant and nonresistant organisms demonstrated an $89 \%$ initial infection control rate with $96 \%$ after further surgery [3]. Analysis of a subset of patients infected with only nonresistant organisms $(n=93)$ revealed an $88 \%$ initial infection control rate and $96 \%$ final rate. Nevertheless, because the two cohorts in the two studies could potentially be different because of other dissimilarities such as comorbidities, it is difficult to make a direct comparison between the two studies. Nevertheless, our data show there is a trend toward a higher risk of failure when the infection is caused by resistant infection.

Our patients had lower mean quality-of-life outcome scores than those previously reported for patients after twostage PROSTALAC ${ }^{\circledR}$ for both of nonresistant and resistant organisms [3]. Again, this difference may be related to other dissimilarities between the two cohorts such as comorbidities.

Lower infection control rates after MRSA and MRSE infections may be related to the difficulty in treating these organisms with local antibiotic therapy (antibiotic-loaded cement). Because $\beta$-lactams are not adequately effective against these organisms, vancomycin is often used. Vancomycin, however, has inferior anti-Staphylococcal activity and tissue penetration compared with $\beta$-lactams [23]. Administration of adjuvant systemic therapy with vancomycin has been advocated, with rifampin being a common choice [21, 27]. Rifampin was used in eight patients in our series, one of whom failed treatment. Given the small numbers, we are unable to report clinical importance with respect to antibiotic selection. 


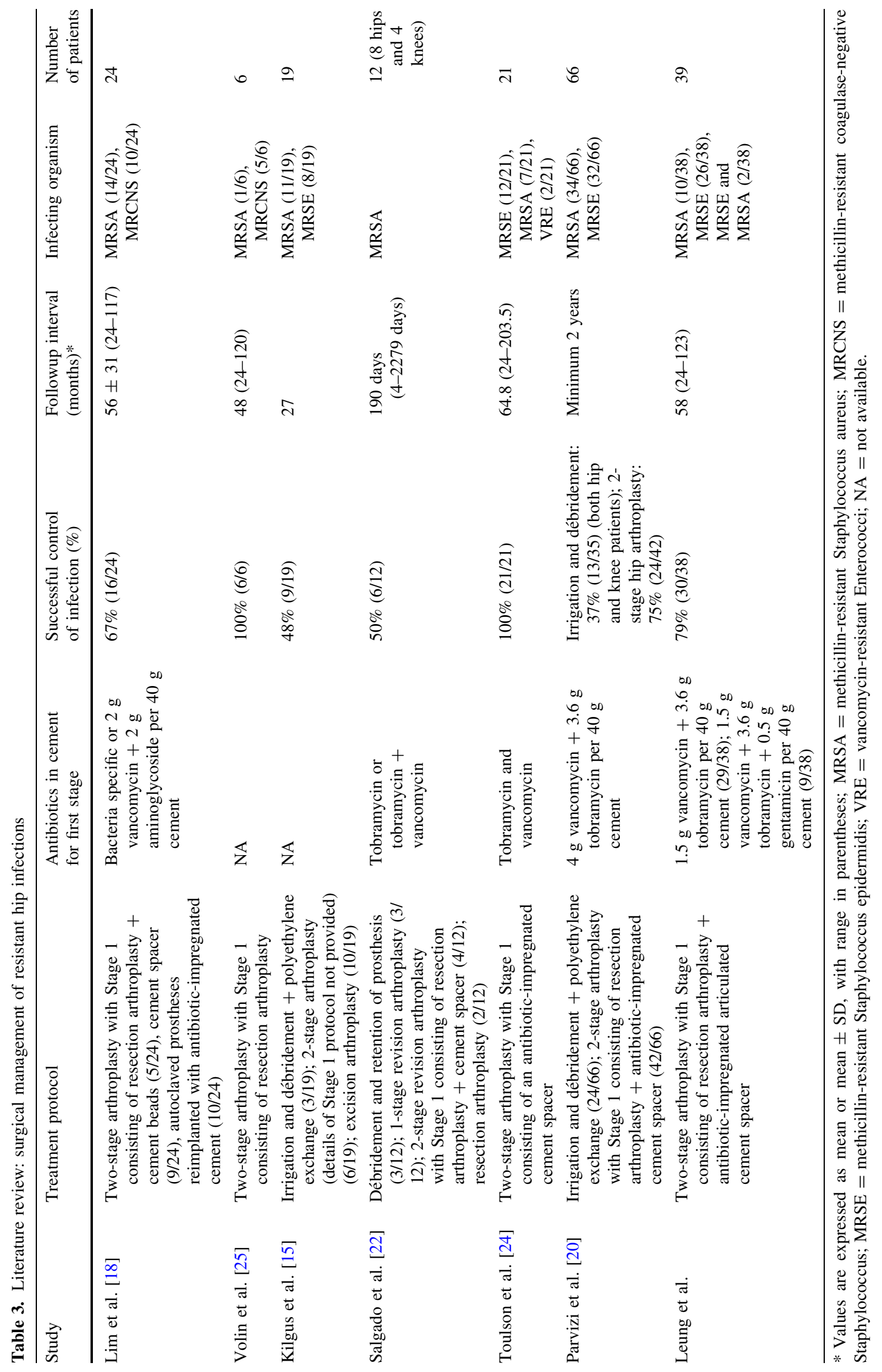


We believe two-stage reconstruction using the PROSTALAC $^{\circledR}$ system is the treatment of choice for periprosthetic infections involving resistant organisms. We found a successful control of infection rate of $79 \%$ at minimum 2-year followup, which is lower than that reported in the literature after treatment of nonresistant organisms. Clearly it is imperative to prevent infection secondary to this group of organisms and a need to develop and evaluate other approaches to the management of this challenging group of patients.

Acknowledgment We thank Dr Nelson V. Greidanus for contributing patients to this study.

\section{References}

1. Amstutz HC, Thomas BJ, Jinnah R, Kim W, Grogan T, Yale C. Treatment of primary osteoarthritis of the hip: a comparison of total joint and surface replacement arthroplasty. $J$ Bone Joint Surg Am. 1984;66:228-241.

2. Bellamy N, Buchanan WW, Goldsmith $\mathrm{CH}$, Campbell J, Stitt LW. Validation study of WOMAC: a health status instrument for measuring clinically important patient relevant outcomes to antirheumatic drug therapy in patients with osteoarthritis of the hip or knee. J Rheumatol. 1988;15:1833-1840.

3. Biring GS, Kostamo T, Garbuz DS, Masri BA, Duncan CP. Twostage revision arthroplasty of the hip for infection using an interim articulated Prostalac hip spacer: a 10- to 15-year followup study. J Bone Joint Surg Br. 2009;91:1431-1437.

4. Buchholz HW, Elson RA, Engelbrecht E, Lodenkamper H, Rottger J, Siegel A. Management of deep infection of total hip replacement. J Bone Joint Surg Br. 1981;63:342-353.

5. Callaghan JJ, Katz RP, Johnston RC. One-stage revision surgery of the infected hip: a minimum 10-year followup study. Clin Orthop Relat Res. 1999;369:139-143.

6. Crockarell JR, Hanssen AD, Osmon DR, Morrey BF. Treatment of infection with debridement and retention of the components following hip arthroplasty. J Bone Joint Surg Am. 1998;80: 1306-1313.

7. Dawson J, Fitzpatrick R, Carr A, Murray D. Questionnaire on the perceptions of patients about total hip replacement. J Bone Joint Surg Br. 1996;78:185-190.

8. Duncan CP, Beauchamp C. A temporary antibiotic-loaded joint replacement system for management of complex infections involving the hip. Orthop Clin North Am. 1993;24:751-759.

9. Garvin KL, Hanssen AD. Infection after total hip arthroplasty: past, present, and future. J Bone Joint Surg Am. 1995;77:15761588.

10. Goulet JA, Pellicci PM, Brause BD, Salvati EM. Prolonged suppression of infection in total hip arthroplasty. J Arthroplasty. 1988;3:109-116.

11. Haleem AA, Berry DJ, Hanssen AD. Mid-term to long-term followup of two-stage reimplantation for infected total knee arthroplasty. Clin Orthop Relat Res. 2004;428:35-39.
12. Hirakawa K, Stulberg BN, Wilde AH, Bauer TW, Secic M. Results of 2-stage reimplantation for infected total knee arthroplasty. J Arthroplasty. 1998;13:22-28.

13. Kantor GS, Osterkamp JA, Dorr LD, Fischer D, Perry J, Conaty JP. Resection arthroplasty following infected total hip replacement arthroplasty. J Arthroplasty. 1986;1:83-89.

14. Katz JN, Phillips CB, Baron JA, Fossel AH, Mahomed NN, Barrett J, Lingard EA, Harris WH, Poss R, Lew RA, Guadagnoli E, Wright EA, Losina E. Association of hospital and surgeon volume of total hip replacement with functional status and satisfaction three years following surgery. Arthritis Rheum. 2003;48: $560-568$.

15. Kilgus DJ, Howe DJ, Strang A. Results of periprosthetic hip and knee infections caused by resistant bacteria. Clin Orthop Relat Res. 2002;404:116-124.

16. Kostuik J, Alexander D. Arthrodesis for failed arthroplasty of the hip. Clin Orthop Relat Res. 1984;188:173-182.

17. Lieberman JR, Callaway GH, Salvati EA, Pellicci PM, Brause BD. Treatment of the infected total hip arthroplasty with a twostage reimplantation protocol. Clin Orthop Relat Res. 1994;301: 205-212.

18. Lim SJ, Park JC, Moon YW, Park YS. Treatment of periprosthetic hip infection caused by resistant microorganisms using 2-stage reimplantation protocol. J Arthroplasty. 2009;24:12641269.

19. Masri BA, Panagiotopoulos KP, Greidanus NV, Garbuz DS, Duncan CP. Cementless two-stage exchange arthroplasty for infection after total hip arthroplasty. J Arthroplasty. 2007;22:72-78.

20. Parvizi J, Azzam K, Ghanem E, Austin MS, Rothman RH. Periprosthetic infection due to resistant staphylococci: serious problems on the horizon. Clin Orthop Relat Res. 2009;467: 1732-1739.

21. Saginur R, Stdenis M, Ferris W, Aaron SD, Chan F, Lee C, Ramotar K. Multiple combination bactericidal testing of staphylococcal biofilms from implant-associated infections. Antimicrob Agents Chemother. 2006;50:55-61.

22. Salgado CD, Dash S, Cantey JR, Marculescu CE. Higher risk of failure of methicillin-resistant Staphylococcus aureus prosthetic joint infections. Clin Orthop Relat Res. 2007;461:48-53.

23. Shams WE, Rapp RP. Methicillin-resistant staphylococcal infections: an important consideration for orthopedic surgeons. Orthopedics. 2004;27:565-568.

24. Toulson C, Walcott-Sapp S, Hur J, Salvati E, Bostrom M, Brause B, Westrich GH. Treatment of infected total hip arthroplasty with a 2-stage reimplantation protocol: update on 'our institution's' experience from 1989 to 2003. J Arthroplasty. 2009;24:10511060.

25. Volin SJ, Hinrichs SH, Garvin KL. Two-stage reimplantation of total joint infections: a comparison of resistant and non-resistant organisms. Clin Orthop Relat Res. 2004;427:94-100.

26. Ware JE Jr, Sherbourne CD. The MOS 36-item short-form health survey (SF-36). I. Conceptual framework and item selection. Med Care. 1992;30:473-483.

27. Widmer AF, Gaechter A, Ochsner PE, Zimmerli W. Antimicrobial treatment of orthopedic implant-related infections with rifampin combinations. Clin Infect Dis. 1992;14:1251-1253.

28. Younger AS, Duncan CP, Masri BA, McGraw RW. The outcome of two-stage arthroplasty using a custom-made interval spacer to treat the infected hip. J Arthroplasty. 1997;12:615-623. 